\title{
Deep Convolutional Neural Networks for Brain tumor segmentation: boosting performance using deep transfer learning: preliminary results
}

\author{
Mostefa Ben naceur ${ }^{1,2}{ }^{\star}$, Mohamed Akil ${ }^{1}$, Rachida Saouli ${ }^{2}$, and Rostom \\ Kachouri $^{1}$ \\ 1 Gaspard Monge Computer Science Laboratory, A3SI, ESIEE Paris, CNRS, \\ University Paris-Est, France. \\ mostefa.bennaceur@esiee.fr, mohamed.akil@esiee.fr, rostom.kachouri@esiee.fr \\ 2 Smart Computer Sciences Laboratory, Computer Sciences Department, Exact.Sc, \\ and SNL, University of Biskra, Algeria. \\ rachida.saouli@esiee.fr
}

\begin{abstract}
Brain tumor segmentation through MRI images analysis is one of the most challenging issues in medical field. Among these issues, Glioblastomas (GBM) invade the surrounding tissue rather than displacing it, causing unclear boundaries, furthermore, GBM in MRI scans have the same appearance as Gliosis, stroke, inflammation and blood spots. Also, fully automatic brain tumor segmentation methods face other issues such as false positive and false negative regions. In this paper, we present new pipelines to boost the prediction of GBM tumoral regions. These pipelines are based on 3 stages, first stage, we developed Deep Convolutional Neural Networks (DCNNs), then in second stage we extract multi-dimentional features from higher-resolution representation of DCNNs, in third stage we developed machine learning algorithms, where we feed the extracted features from DCNNs into different algorithms such as Random forest (RF) and Logistic regression (LR), and principal component analysis with support vector machine (PCA-SVM). Our experiment results are reported on BRATS-2019 dataset where we achieved through our proposed pipelines the state-of-the-art performance. The average Dice score of our best proposed brain tumor segmentation pipeline is $0.85,0.76,0.74$ for whole tumor, tumor core, and enhancing tumor, respectively. Finally, our proposed pipeline provides an accurate segmentation performance in addition to the computational efficiency in terms of inference time makes it practical for day-to-day use in clinical centers and for research.
\end{abstract}

Keywords: Brain Tumor Segmentation. Convolutional Neural Networks. Support Vector Machine. Glioblastomas · Transfer Learning · Principal Component Analysis.

\footnotetext{
* Corresponding author.
} 


\section{Introduction}

Brain tumor is a growing abnormal cell in the brain or central spin canal [1]. Usually, a radiologist uses MRI scans as the most effective [2] technique to generate Multi-modal images and to identify different tumor regions in the soft tissues of the brain. In general, a radiologist generates four standard MRI images modalities for Gliomas tumors diagnosis [3]: T2-weighted fluid attenuated inversion recovery (Flair), T1-weighted (T1), T1-weighted contrast-enhanced (T1c), and T2-weighted (T2) for each patient. Furthermore, what makes the diagnosis hard for radiologist is that each patient has a different health condition, age, gender, in addition to Glioblastima tumors is unexpected, in other words, these tumors could appear anywhere in the brain.

Current state-of-the-art methods in the field of computer vision are based on a deep learning, especially Convolutional Neural Networks (CNNs). Where in CNNs [4], we find a feature extractor with a bank of convolution layers, then pooling layers to make the images less sensitive and invariant to small translations, then the last step in CNNs is a classifier (in general Softmax layer) that classifies each pixel into one of a set of classes. After the breakthrough in 2012 of AlexNet [5] model that outperformed the state-of-the-art methods in the field of object recognition, many methods obtained high results in many fields especially in medical field such as [6], [7], [8], [11], [9], [10]. In general, these methods are trained on 4 types of MRI images: Flair, T1, T1c, and T2.

Our ongoing work is based on our previous work [9], [15]. In this paper, we are focusing on two major issues: (1) false positive regions - where the model predicts non-tumor regions as tumor regions but in fact they are not-, (2) false negative regions - where the model classifies some regions as non-tumor regions but in fact they are. In [9], [15] we addressed the problem of false positive regions by two steps: we used a global threshold for each slice to remove small non-tumoral regions based on connected-components, then in second step, to enhance the post-processing step more we used a morphological opening operator. Despite the success of these two post-processing steps, further steps are required to improve the segmentation results. The main reason of these two issues (i.e. false positive regions and false negative regions) is the classifier of DCNNs, where in our case the classifier is the Softmax function. Softmax function gives an estimated vector at the end after each forward propagation of DCNNs, by normalizing the outputs to stay between 0 and 1, i.e., the outputs become as probabilities. Then, we pick the result of the forward pass based on the maximum probability among all probabilities, and this maximum probability represent a class, in our case, one class out of the 4 predefined classes (i.e., Necrotic and Non-Enhancing tumor, Peritumoral Edema, Enhancing tumor and healthy tissue). The Softmax function is a simple and an accurate function for training phase, but it is not adequate for the prediction or test phase for the problem of instance segmentation. The extracted features from MRI scans are complicated and in this case of DCNNs are hierarchical, so, classifying these features among a set of classes is not simple and intuitive for Softmax function. To overcome this issue, we developed two brain tumor segmentation pipelines, firstly, we extract 
feature maps from DCNNs, secondly, these features maps become the dataset of training and testing for another machine learning algorithms. The first pipeline is based on two algorithms: RF and LR, where the second pipeline is based on PCA-SVM.

The aim of this paper is to propose and develop new pipelines for brain tumor segmentation. Where we use the technique of transfer learning to extract features from DCNNs architecture then we feed these features into another machine learning algorithms (i.e., RF, LR and PCA-SVM), then for the first pipeline we combine the results of DCNNs, RF and LR into one 3D image, while for the second pipeline we train PCA-SVM on the extracted features maps. The proposed pipelines are used to segment the brain tumors of GBM with both high- and low-grade.

\section{Proposed Method}

In this paper, we proposed two pipelines to boost the segmentation performance of GBM brain tumor. One of the main issues for segmentation performance degradation is false positive and false negative regions, and the main reason of these issues is Softmax function, where this simple function does not provide an accurate results at the prediction phase (testing phase). Thus, to improve the accuracy of Softmax in the first proposed pipeline, we combine the results of Softmax with the results of two algorithms (.i.e., RF, LR), while for the second pipeline, we feed the feature maps of the last layer of DCNNs to PCA-SVM. So for both pipelines, the first step, we extract the higher-resolution feature maps of the last layer before output layer (Softmax layer). The second step, we prepare these feature maps to become in a better representation for machine learning algorithms.

\subsection{Features visualization}

In this paper, we trained a DCNNs model from scratch where this model is trained for brain tumor segmentation of GBM. DCNNs work by learning to extract hierarchical features from images. The algorithm of CNNs [4] is originally inspired by the visual system. In 1962, Hubel and Wiesel [16] discovered that each type of neurons in the visual system, responds to one specific feature: vertical lines, horizontal lines, shapes, etc. From this discovery, the algorithm of CNNs was developed. Thus, CNNs detect low-level features such as lines, edges, etc. and high-level features such as shapes and objects. These hierarchical features help CNNs algorithm to better locate the boundaries of the tumor regions (see Fig.1). The figure 1 shows different features of a subject from our training set, as you can see, we can clearly distinguish the boundaries of each region. 

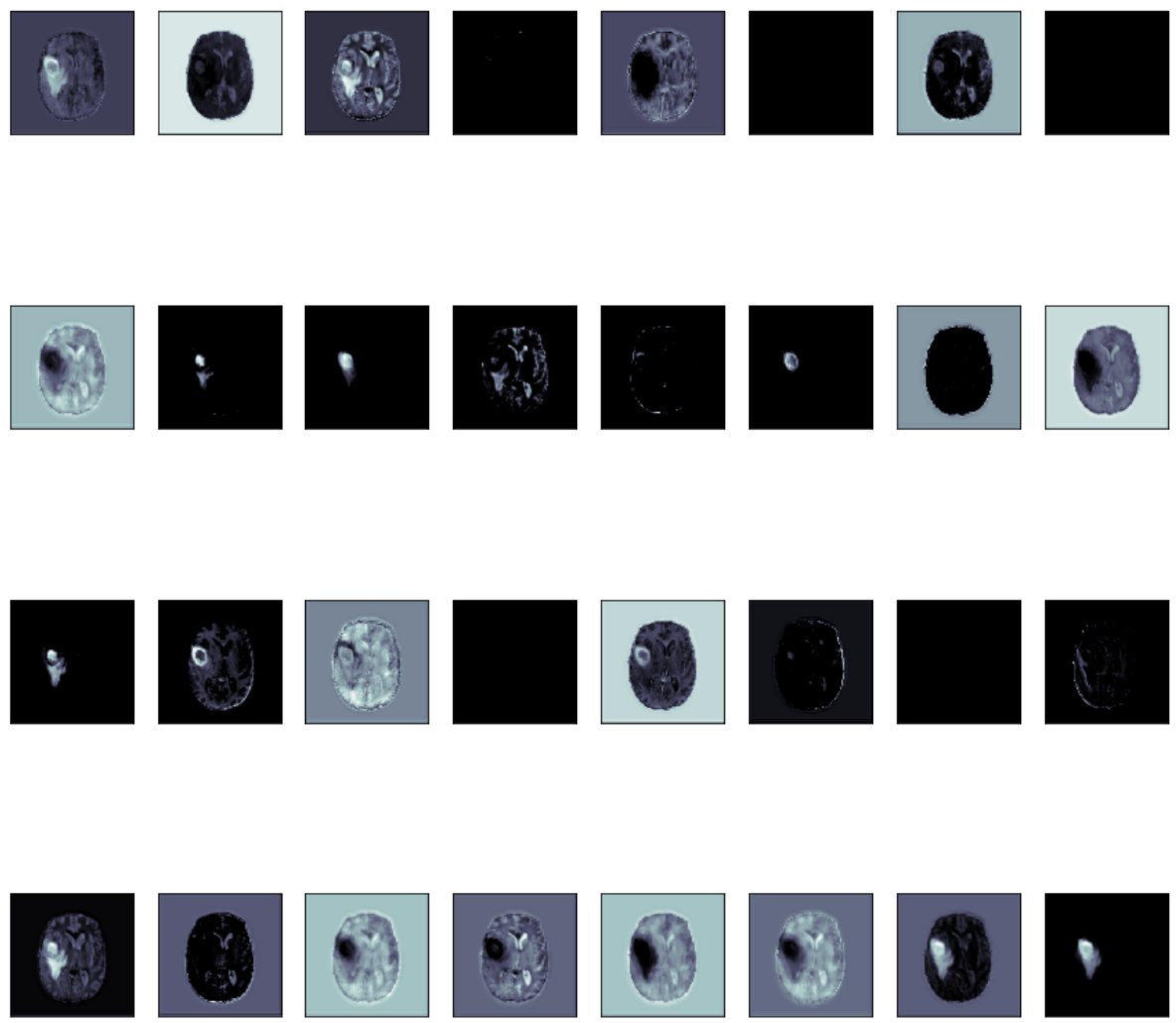

Fig. 1: Features visualization of the layer 16 of the CNNs model. Layer 16 of CNNs has 32 features maps, each has a filter to search for a specific feature the most representative in a MRI image

\subsection{Fusion solution-based predictions aggregation}

The flowchart for detecting GBM brain tumors (see Fig.2) is composed of two parts: the first part is composed of the DCNNs model and the second part is composed of machine learning algorithms such as RF and LR. To integrate the LR and RF in DCNNs, we first extracted the feature maps of the last layer before the output, then we replaced the Softmax layer with the aforementioned algorithms (i.e., LR and RF). Then we fused the results of these three algorithms (LR, RF and Softmax) into one result to diagnose the presence of tumor in each extracted feature maps and to diagnose the class of this tumor in each pixel.

To develop a DCNNs architecture, we have either pixel-wise approach or patch-wise approach. The first approach deals with pixels, while second approach deals with patches. In this paper, we used patch-wise classification, car it provides a good segmentation results [19], [20], [21] compared to pixel-wise classification, 


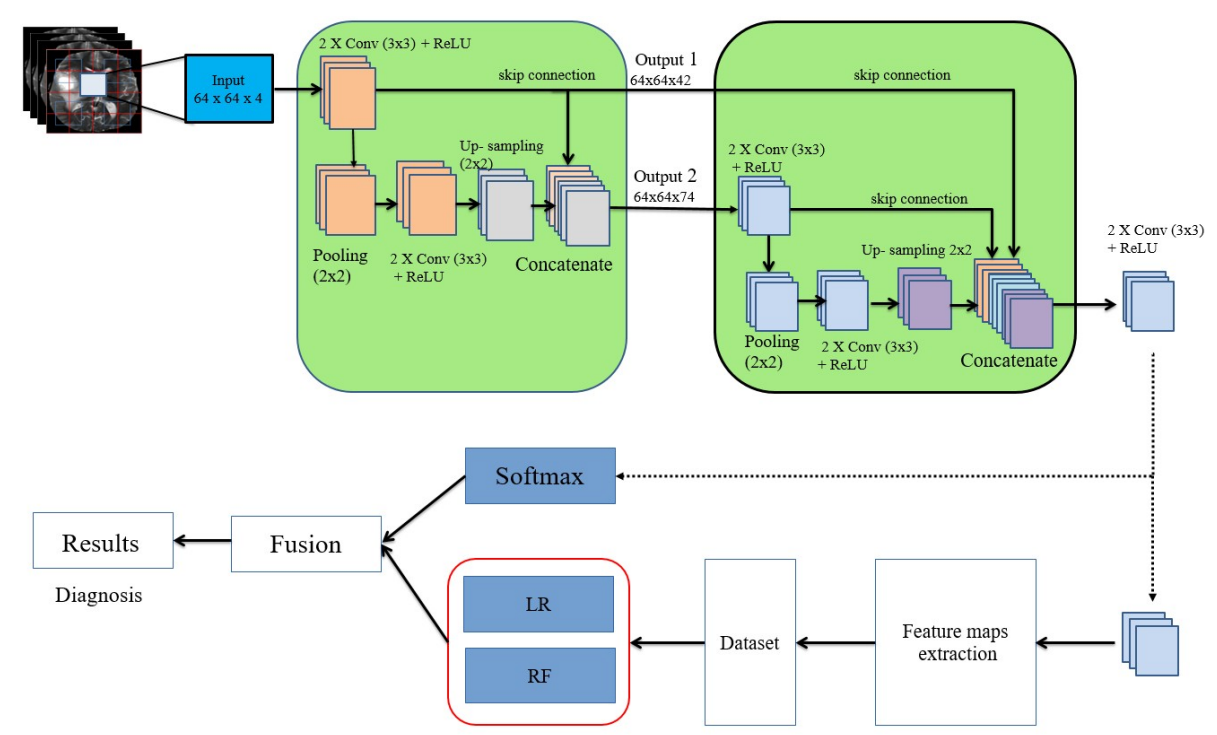

Fig. 2: Flowchart fusion solution for predicting GBM brain tumors. This flowchart has two parts: the first part (two green boxes) represent the DCNNs model, the second part (three blue boxes) represent the aggregation results of Sofmtax, LR and RF in a single prediction result

in addition it is less prone to overfitting, and these advantages are due to the parameters sharing between neurons in the network. Patch-wise approach takes as an input patches with limited size, and after extensive experiments to get the best patch's size (e.g., $32 \times 32 \times 4,64 \times 64 \times 4$ ) for our approach, we observed that patches with size $(64 \times 64 \times 4)$ provide an accurate segmentation performance in terms of the evaluation metrics (see section 2.6). The optimization of DCNNs is done using stochastic gradient descent with mini-batch size equals to 8 and learning rate computed as follows:

$$
L E R_{i}=10^{-3} \times 0.99^{L E R_{i-1}}
$$

Where the initial learning rate (LER) was $L E R_{0}=0.001, L E R_{i}\left(i \in \mathbb{N}^{+}\right)$ is the new learning rate, $L E R_{i-1}$ is the learning rate of the last epoch, 0.99 is a decreasing factor. The DCNNs model was implemented on Keras which is a high-level open source Deep Learning library, and Theano as a Back-end, where Theano exploits GPUs to optimize Deep Learning architectures (i.e., to minimize the error). In this work, all our results are obtained using Python environment on windows 64-bit, Intel Xeon processor $2.10 \mathrm{GHz}$ with $24 \mathrm{~GB}$ RAM, and the training is done on Nvidia Quadro GPU K1200 with 4 GB RAM.

After training DCNNs model, we extracted feature maps of the last layer before applying the Softmax classifier to train two other classifiers: LR, RF. These two classifiers are powerful to avoid the overfitting problem, in addition, RF is 


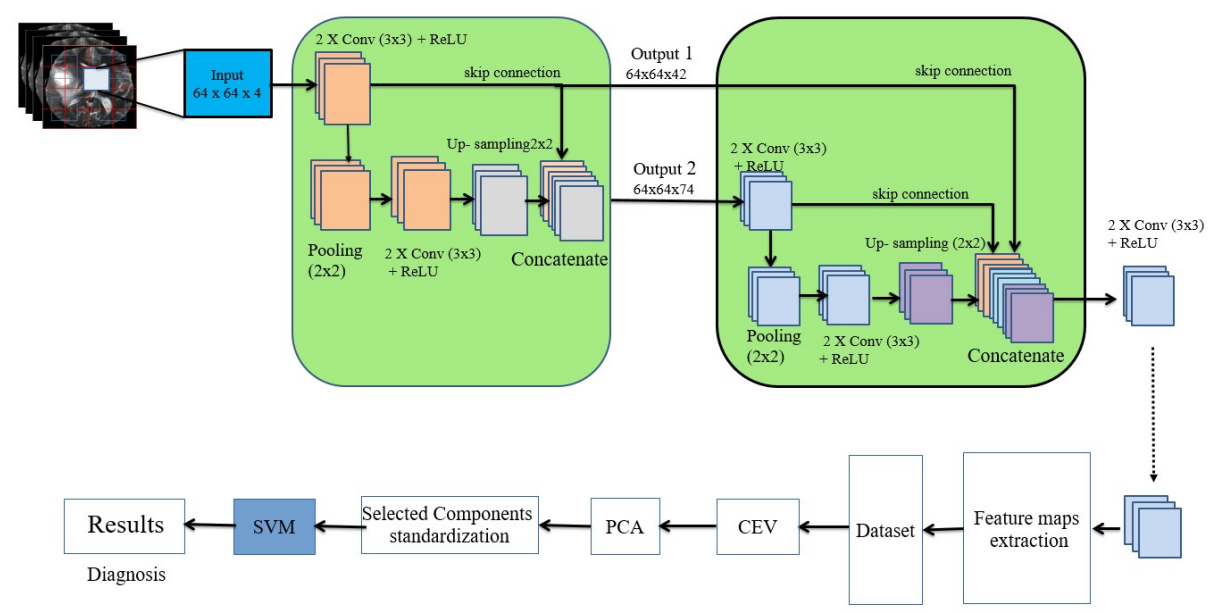

Fig. 3: Flowchart of PCA-SVM solution for predicting GBM brain tumors. This flowchart starts by a DCNNs model (two green boxes), then the extracted feature maps from DCNNs will be reduced by applying cumulative explained variance (CEV), and the reduced selected components are fed into SVM to predict the class of each pixel in GBM brain tumor.

considered as an ensemble learning, where it is used to improve the system's performance in many applications.

\subsection{Semi automatic-based Support vector machine}

In the flowchart in Fig.3, we used an SVM method to segment the MRI images of patients with GBM. In this flowchart, we first trained DCNNs as we did in the first step (see section 2.1). To improve the segmentation performance, we extracted and collected the feature maps of the last layer before the classifier (Softmax) into one dataset for training and testing (60\% and $40 \%$, respectively). Secondly, to reduce the huge dimensionality of the features maps, we computed the cumulative explained variance (CEV) in order to obtain the number of components that cover all variance (most useful information). By using these techniques, we can reduce the number of redundant features in addition to noise, because noise do not have a high variance allow it to be extracted among the first components. After applying CEV method on the extracted features, we observed that $99 \%$ of variances are concentrated on only 139 components out of 3200 components; which means that 3061 of components hold redundant features and noise (see Fig.4).

As you can see from Fig.4, the first figure (4.a) represents the dimension of all features, where (4.b) represents only 139 dimensions among 3200 dimensions, thus from these two figures we can conclude that 139 componenents represent $99 \%$ of variances (information). After computing CEV, we applied principal 


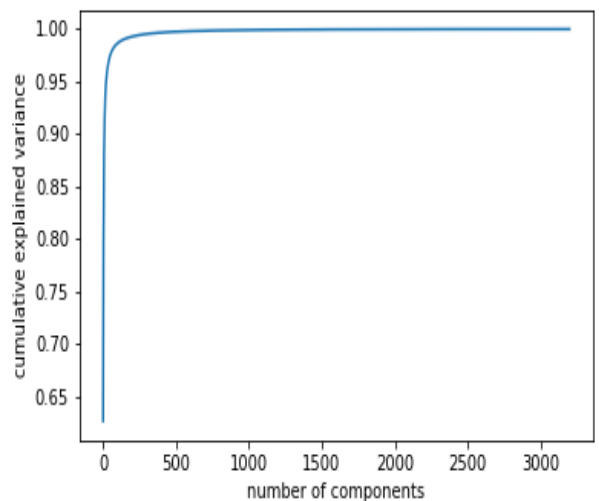

(a) all components (all dimensions)

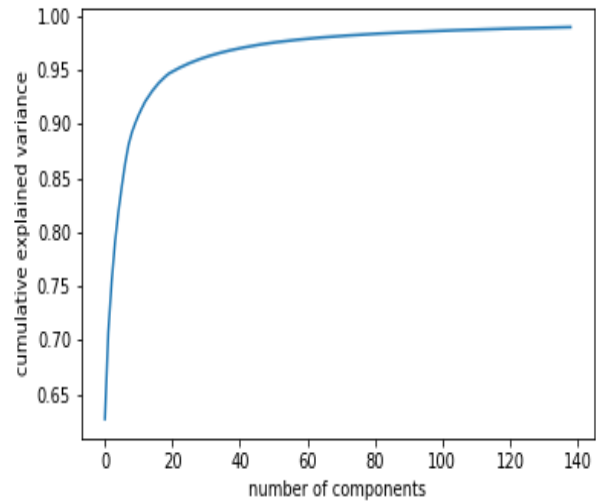

(b) 139 components (139 dimensions)

Fig. 4: Cumulative explained variance curve for (a) all components and for (b) 139 components. It can be seen that we only need 139 components out of 3200 components to represent $99 \%$ of the features. The remaining components (i.e., 3061) represent redundant features and noise.

components analysis (PCA) to reduce the dimensionality of features until 139 components; for each patch, we use only 139 components instead of 3200 components. Reducing the dimensionality using PCA helps to get new features more representative, without getting redundant features and noise which need a lot of preprocessing to remove them.

The last step in this flowchart (see Fig.3) consists of applying SVM which is one of the most powerful methods for classification as it can deal with many forms of data and classification problems (binary, multi, linear, non-linear). To develop a SVM method: firstly, we need a lot of data, here we use the computed components by using PCA. Secondly, we need to specify the type of problem, in our case, it is a multi-classification issue (4 classes). Usually, multi-classification problem is a non linearly separable issue, so to verify the type of problem (linear, non-linear), we draw the first three components in 3D space (see Fig.5):

As you can see in figure (5.a and 5.b), we drew only 3 components from 139 components, and as expected the issue of multi-classification in our case is non linearly separable. From figure 5, we conclude that the issue that we are dealing with, is multi-classification and non linearly separable issue, thus this conclusion helps us to determine the hyperparameters of SVM especially the kernel. In general, in SVM, there are three types of kernels: linear, polynomial and radial basis function (RBF). Firstly we can eliminate linear kernel because it is used for linearly separable issues, secondly polynomial kernel is computionally expensive and needs a lot of memory, thus, in this paper we use the RBF kernel. Moreover, for the other hyperparameters: coefficient gamma $\gamma$ and $\mathrm{C}$ slack-penalty: because SVM is sensitive to outliers and feature scaling and as we mentioned earlier the problem of multi-classification of brain tumor segmentation is non linearly 


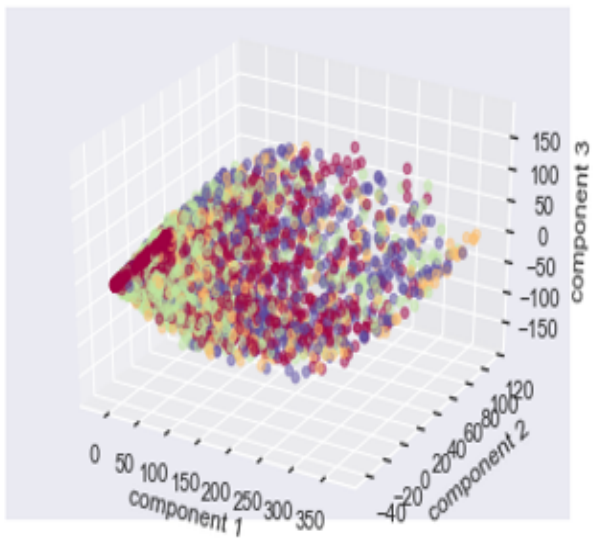

(a)

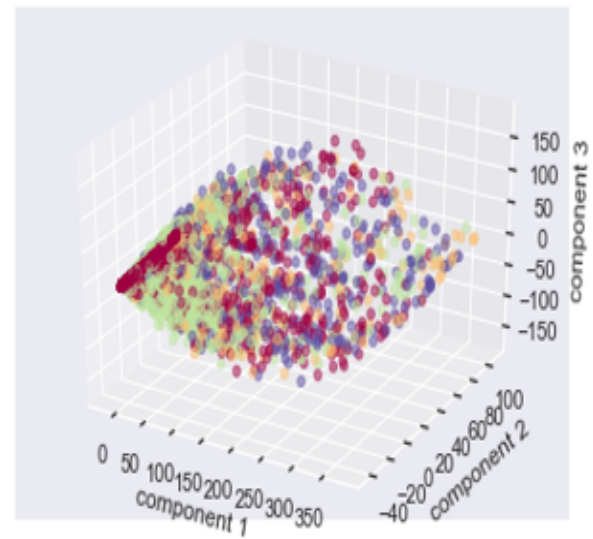

(b)

Fig. 5: Scatter plot for the first three components in 3D space in (a) training and (b) testing datasets. It can be seen that in the training and testing dataset, the issue of brain tumor segmentation is a nonlinear multi classification issue. Best viewed in color.

seperable issue which means in this case $\mathrm{C}$ slack-penelity is great than zero (soft-margin classification); some instances could be on the street of the decision boundary (margin violations). Furthermore, the value of gamma $\gamma$ controls the influence of each feature in its search space, thus in this case and because of outliers, gamma $\gamma$ should be a little bit high. Because there is no straightforward method to select gamma $\gamma$ and $\mathrm{C}$, we used cross-validation with random search and grid search techniques on a small dataset of 30 brain images from the training set. The result of cross-validation as the following: gamma $\gamma=0.001$ and $\mathrm{C}=5$. Moreover, SVM is sensitive to feature scaling because the kernel RBF is used to compute the objective function of SVM, thus, RBF assumes that all features are centered around zero and the variance's magnitude for all features is the same, so, we standardized the computed components of PCA, to be centered with 0 mean and a standard deviation of 1 as the following (see equation 2):

$$
\operatorname{Standardization}(X)=\frac{X-U}{\sigma}
$$

Where $\mathrm{X}$ is a sample, $\mathrm{U}$ is the Mean of the training set and $\sigma$ is the Standard Deviation.

Brain tumor segmentation is primarily used for diagnosis, treatment, and follow-up. The developed pipeline in this paper is applied to GBM which are brain tumors and life-threating deseases. These tumors have four classes: Necrotic and Non-Enhancing tumor, Peritumoral Edema, Enhancing tumor and healthy tissue. To interpret MRI images, a radiologist employs a manual segmentation. 
Furthermore, it is known that the manual segmentation in MRI images is a time-consuming and a tedious procedure. In general, there are three methods to obtain a brain tumor segmentation image: manual, semi-automatic and fully automatic. In this paper, we investigate the segmentation performance using extracted features from DCNNs architecture and a SVM method which is a supervised learning method. Our approach is semi-automatic, where this approach needs a user interaction to reduce the misclassified regions by the SVM method, that are in most cases false positives.

\subsection{Dataset}

We have used a publicly available dataset called BraTS 2019 dataset [12], [13], [14], [17], [18], where the training set has 259 patient's brain images with highgrade (HGG) and 76 patient's brain images with low-grade (LGG). Each patient's brain image comes with 4 MRI sequences (i.e., Flair, T1, T1c and T2) and the ground truth of 4 segmentation labels which are obtained manually by radiologists: Healthy tissue, Necrotic and Non-Enhancing tumor, Peritumoral Edema, Enhancing core. BRATS 2019 validation and testing sets contain 125 and 166 images respectively of patients with unknown grade, i.e. the validation and testing sets do not have the Ground Truth labels. Our DCNNs model is built upon 2D image patches (Slice), where this model predicts the pixel's class which is the center of the $2 \mathrm{D}$ patch.

\subsection{Pre-processing}

To enhance the quality of the MRI scans and to remove some noise, we applied 3 steps:

1. Removing $1 \%$ highest and lowest intensities: this technique helps to remove some noise at the tail of the histogram, where this step has provided good results in many research [11].

2. Subtracting the mean and dividing by the standard deviation of non-zero values in all channels: this technique is used to center and to put the data in the same scale, i.e. bringing the mean intensity value and the variance between one and minus one.

3. In this step, we try to isolate the background from the tumoral regions by assigning the minimum values to -9 , where it has been observed that using integer numbers between -5 to -15 , fit our DCNNs model. The application of the second pre-processing step, led to bringing the mean value in the range $[-1,1]$, in other words, the intensities of all regions in addition to healthy and background became between -1 and 1 . As we know, the intensity of background pixels of MRI images in BRATS data equals to 0, thus to isolate the zero pixels (background) from the other regions, we normalized the histogram of the MRI images by shifting the zero pixels to another bin outside the range $[-1,1]$. We found that the bin -9 in many experiments, gives good results in the training and testing phases. 


\subsection{Evaluation}

To evaluate the performance of the proposed flowchart (see Fig.2), we used BRATS online evaluation system ${ }^{3}$. This system evaluates the uploaded images using four metrics: Dice score, Sensitivity, Specificity, and Hausdorff distance: Dice $(\mathrm{P}, \mathrm{T})=\frac{\left|P_{1} \wedge T_{1}\right|}{\left(\left|P_{1}\right|+\left|T_{1}\right|\right) / 2}, \quad$ Sensitivity $(\mathrm{P}, \mathrm{T})=\frac{\left|P_{1} \wedge T_{1}\right|}{\left|T_{1}\right|}, \quad$ Specificity $(\mathrm{P}, \mathrm{T})$ $=\frac{\left|P_{0} \wedge T_{0}\right|}{\left|T_{0}\right|}$,

$$
\text { Hausdorff }(\mathrm{P}, \mathrm{T})=\max \left\{\sup _{p \in \partial P_{1}} \inf _{t \in \partial T_{1}} d(p, t), \sup _{t \in \partial T_{1}} \inf _{p \in \partial P_{1}} d(t, p)\right\}
$$

\section{$3 \quad$ Results}

In this section, we evaluate our proposed brain tumor segmentation pipelines on a public BRATS 2019 dataset using the online evaluation system.

Table 1: Evaluation results of fusion solution-based predictions aggregation pipeline on BRATS 2019 validation set. WT, TC, ET denote whole tumor, tumor core, enhancing tumor, respectively.

\begin{tabular}{|l|c|c|c|c|c|c|c|c|c|c|c|c|c|c|c|}
\hline & \multicolumn{3}{|c|}{ Dice score } & \multicolumn{2}{|c|}{ Sensitivity } & \multicolumn{3}{c|}{ Specificity } & \multicolumn{3}{c|}{ Hausdorff } \\
\cline { 2 - 11 } & WT & TC & ET & WT & TC & ET & WT & TC & ET & WT & TC & ET \\
\hline Mean & 0.84 & 0.70 & 0.61 & 0.84 & 0.71 & 0.69 & 0.99 & 0.99 & 1.0 & 22.64 & 20.40 & 13.76 \\
Standard deviation & 0.13 & 0.23 & 0.33 & 0.16 & 0.26 & 0.28 & 0.01 & 0.01 & 0.01 & 26.10 & 24.63 & 24.90 \\
Median & 0.88 & 0.77 & 0.77 & 0.89 & 0.80 & 0.81 & 0.99 & 1.0 & 1.0 & 8.37 & 10.68 & 3.16 \\
25 quantile & 0.84 & 0.58 & 0.43 & 0.82 & 0.61 & 0.60 & 0.99 & 0.99 & 1.0 & 3.61 & 6.40 & 2.0 \\
75 quantile & 0.91 & 0.87 & 0.86 & 0.94 & 0.92 & 0.89 & 1.0 & 1.0 & 1.0 & 37.29 & 22.20 & 11.0 \\
\hline
\end{tabular}

Table 2: Evaluation results of fusion solution-based predictions aggregation pipeline on BRATS 2019 testing set. WT, TC, ET denote whole tumor, tumor core, enhancing tumor, respectively.

\begin{tabular}{|l|c|c|c|c|c|c|}
\hline \multirow{2}{*}{} & \multicolumn{3}{|c|}{ Dice score } & \multicolumn{3}{c|}{ Hausdorff } \\
\cline { 2 - 7 } & WT & TC & ET & WT & TC & ET \\
\hline Mean & 0.84709 & 0.75889 & 0.73703 & 12.99701 & 15.4957 & 6.03933 \\
Standard deviation & 0.15312 & 0.25993 & 0.23841 & 23.97851 & 25.62727 & 16.45033 \\
Median & 0.89588 & 0.85913 & 0.8148 & 4.30077 & 8.09315 & 2.23607 \\
25 quantile & 0.83621 & 0.74323 & 0.70943 & 3 & 4 & 1.41421 \\
75 quantile & 0.92368 & 0.9143 & 0.87902 & 7.95064 & 14.65374 & 3.74166 \\
\hline
\end{tabular}

\footnotetext{
${ }^{3}$ https://ipp.cbica.upenn.edu/
} 
Table 1 and Table 2 show the segmentation results of our proposed pipeline (see Fig.2) for fully automatic brain tumor segmentation. The prediction of tumoral regions is performed using $2 \mathrm{D}$ patches with size equals to $64 \times 64 \times 4$ (4 corresponds to using different modalities such as T1, post-contrast T1, T2 and FLAIR). Then, we extract the feature maps of the last layer before Softmax function, then we feed these features into different machine learning algorithms such as RF and LR. Last step, we combine the results of DCNNs, RF and LR into one 3D image using voting technique; where the most predicted label among the predictions (e.g., 1, 1 and 0) of these classifiers (algorithms) become the result (in this case the label becomes 1). Table 1 and Table 2 shows the validation and testing scores, and as you can see, we are able to achieve segmentation results comparable to the top performing methods in state-of-the-art such as the work of [10]. Moreover, the achieved median score is high: 0.88, 0.89 for whole tumor on dice score (validation and testing, respectively), this high values is due to achieving a good segmentation performance for most MRI images.

Table 3: Evaluation results of semi-automatic-based support vector machine pipeline on some subjects of BRATS 2019 training set. WT, TC, ET denote whole tumor, tumor core, enhancing tumor, respectively.

\begin{tabular}{|c|c|c|c|c|c|c|c|c|c|c|c|c|}
\hline & \multicolumn{3}{|c|}{ Dice score } & \multicolumn{3}{c|}{ Sensitivity } & \multicolumn{3}{c|}{ Specificity } & \multicolumn{3}{c|}{ Hausdorff } \\
\cline { 2 - 13 } & WT & TC & ET & WT & TC & ET & WT & TC & ET & WT & TC & ET \\
\hline "BraTS19_2013_10_1" & 1 & 0.58996 & 0.24228 & 1 & 0.74294 & 0.19889 & 1 & 0.96818 & 0.99043 & 0 & 29.03446 & 26.41969 \\
\hline "BraTS19_2013_11_1" & 1 & 0.60334 & 0.14959 & 1 & 0.80945 & 0.25156 & 1 & 0.96026 & 0.97204 & 0 & 13 & 13 \\
\hline "BraTS19_2013_12_1" & 1 & 0.46689 & 0.18173 & 1 & 0.79719 & 0.28464 & 1 & 0.89892 & 0.93791 & 0 & 27.09243 & 30.09983 \\
\hline
\end{tabular}

Table 3 shows the segmentation results of our semi-automatic method that is based on PCA and SVM. In this table, we show differents metrics (see section 2.6). As you can see our method provides good segmentation results especially on tumor core and enhancing tumor regions and that is due to the extracted features from the layer 16 of the DCNNs model and the selected components of PCA. Please note that this method is semi automatic, where it needs a user interaction to select the tumoral regions, that's why the whole tumor is 1 for Dice score, Sensitivity and Specificity, and 0 for Hausdorff distance. Please note also that the validation and testing sets do not have the ground truth labels for the four regions. In the future, we will study the impact of each layer in the DCNNs model in addition to the standardized components of PCA on the segmentation results.

\section{Discussion and Conclusions}

In this paper, we developed two brain tumor segmentation pipelines for GBM brain tumors, these pipelines are based on DCNNs and learned features maps. The proposed DCNNs model model uses skip connections and up-sampling filters 
to maximize the features representation inside the model. Also, using short skip connections helps to complete the missing information during the pooling layers and convolution striding, and long skip connections encourage the feature reuse which assists the model to combine the low-level and the high-level features and to better locate the tumor regions. Moreover, to overcome the issues of false positive and false negative regions, we extracted the feature maps to train another two machine learning algorithms: random forest, logistic regression, and SVM. These algorithms showed a high impact on the segmentation performance.

Our experimental results show that our proposed brain tumor segmentation pipelines improved the evaluation metrics (.i.e., Dice score, Sensitivity, Specificity, Hausdorff). The Mean Dice score of our best proposed fully automatic brain tumor segmentation pipeline (see Fig.2) is 0.85, 0.76, 0.74 for whole tumor, tumor core, and enhancing tumor, respectively. The second pipeline (see Fig.3) is a semi automatic method based on PCA, SVM and learned feature maps of DCNNs. In this study, we used cumulative explained variance with PCA to reduce the dimension of features to 139 components that are enough to provide $99 \%$ of variances for each patient image. Then, we applied a SVM method to predict the class of each pixel. The showing segmentation results are promising and give a high segmentation performance, in which we can enhance it in the future with more investigation in the different phases from feature extraction to prediction using machine learning algorithms. Moreover, the proposed pipeline is suitable for adopting in research and as a part of different clinical settings.

As a perspective of this research, we intend to investigate principal component analysis (PCA) to explore and reduce the features dimensionality, where with this technique we can improve the results by using only the features that have a huge impact on the segmentation results. After this study, we intend to integrate support vector machine (SVM), PCA and DCNNs into an end-to-end supervised learning algorithm.

\section{References}

1. Young, R. J. and Knopp, E. A. (2006). Brain mri: tumor evaluation. Journal of Magnetic Resonance Imaging: An Official Journal of the International Society for Magnetic Resonance in Medicine, 24(4):709-724.

2. Akram, M. U. and Usman, A. (2011). Computer aided system for brain tumor detection and segmentation. In Computer Networks and Information Technology (ICCNIT), 2011 International Conference on, pages 299-302. IEEE.

3. Issin, A., Direkoglu, C., and S,ah, M. (2016). Review of mri-based brain tumor image segmentation using deep learning methods. Procedia Computer Science, 102:317-324.

4. Yann LeCun, Léon Bottou, Yoshua Bengio, Patrick Haffner, et al. Gradientbased learning applied to document recognition. Proceedings of the IEEE, 86(11):2278-2324, 1998.

5. Alex Krizhevsky, Ilya Sutskever, and Geoffrey E Hinton. Imagenet classification with deep convolutional neural networks. In Advances in neural information processing systems, pages 1097-1105, 2012. 
6. Axel Davy, Mohammad Havaei, David Warde-Farley, and T. Lam J. Pierre-Marc C. Aaron L. Hugo P. Chris B. YoshuaBiard, Antoine. Brain tumor segmentation with deep neural networks. in: Proceedings of the MICCAI Workshop on Multimodal Brain Tumor Segmentation Challenge BRATS, pages 01-05, 2014.

7. Sérgio Pereira, Adriano Pinto, Victor Alves, and Carlos A Silva. Deep convolutional neural networks for the segmentation of gliomas in multi-sequence mri. in:Proceedings of the MICCAI Workshop on Multimodal Brain Tumor Segmentation Challenge BRATS, pages 52-55, 2015.

8. Peter D Chang et al. Fully convolutional neural networks with hyperlocal features for brain tumor segmentation. In Proceedings MICCAI-BRATS Workshop, pages 4-9, 2016.

9. Mostefa Ben naceur, Rachida Saouli, Mohamed Akil, and Rostom Kachouri. Fully automatic brain tumor segmentation using end-to-end incremental deep neural networks in mri images. Computer methods and programs in biomedicine, 166:39-49, 2018.

10. Xiaomei Zhao, Yihong Wu, Guidong Song, Zhenye Li, Yazhuo Zhang, and Yong Fan. A deep learning model integrating fcnns and crfs for brain tumor segmentation. Medical image analysis, 43:98-111, 2018.

11. Mohammad Havaei, Axel Davy, David Warde-Farley, Antoine Biard, Aaron Courville, YoshuaBengio, Chris Pal, Pierre-Marc Jodoin, and Hugo Larochelle. Brain tumor segmentation with deep neural networks. Medical image analysis, $35: 18-31,2017$.

12. B. H. Menze, A. Jakab, S. Bauer, J. Kalpathy-Cramer, K. Farahani, J. Kirby, et al. "The Multi-modal Brain Tumor Image Segmentation Benchmark (BRATS)", IEEE Transactions on Medical Imaging 34(10), 1993-2024 (2015) DOI: 10.1109/TMI.2014.2377694

13. S. Bakas, H. Akbari, A. Sotiras, M. Bilello, M. Rozycki, J.S. Kirby, et al., "Advancing The Can-cer Genome Atlas glioma MRI collections with expert segmentation labels and radiomic features", Nature Scientific Data, 4:170117 (2017) DOI: 10.1038/sdata.2017.117

14. S. Bakas, M. Reyes, A. Jakab, S. Bauer, M. Rempfler, A. Crimi, et al., "Identifying the Best Ma-chine Learning Algorithms for Brain Tumor Segmentation, Progression Assessment, and Overall Survival Prediction in the BRATS Challenge", arXiv preprint arXiv:1811.02629 (2018)

15. Mostefa Ben naceur, Kachouri, R., Akil, M., Saouli, R. (2019, June). A New Online Class-Weighting Approach with Deep Neural Networks for Image Segmentation of Highly Unbalanced Glioblastoma Tumors. In International Work-Conference on Artificial Neural Networks (pp. 555-567). Springer, Cham.

16. Hubel, David H., and Torsten N. Wiesel. "Receptive fields, binocular interaction and functional architecture in the cat's visual cortex." The Journal of physiology 160.1 (1962): 106-154.

17. S. Bakas, H. Akbari, A. Sotiras, M. Bilello, M. Rozycki, J. Kirby, et al., "Segmentation Labels and Radiomic Features for the Pre-operative Scans of the TCGA-GBM collection", The Cancer Imaging Archive, 2017. DOI: 10.7937/K9/TCIA.2017.KLXWJJ1Q

18. S. Bakas, H. Akbari, A. Sotiras, M. Bilello, M. Rozycki, J. Kirby, et al., "Segmentation Labels and Radiomic Features for the Pre-operative Scans of the TCGA-LGG collection", The Cancer Imaging Archive, 2017. DOI: 10.7937/K9/TCIA.2017.GJQ7R0EF 
19. Ronneberger, O., Fischer, P., \& Brox, T. (2015, October). U-net: Convolutional networks for biomedical image segmentation. In International Conference on Medical image computing and computer-assisted intervention (pp. 234-241). Springer, Cham.

20. Çiçek, Ö., Abdulkadir, A., Lienkamp, S. S., Brox, T., \& Ronneberger, O. (2016, October). 3D U-Net: learning dense volumetric segmentation from sparse annotation. In International conference on medical image computing and computer-assisted intervention (pp. 424-432). Springer, Cham.

21. Milletari, F., Navab, N., \& Ahmadi, S. A. (2016, October). V-net: Fully convolutional neural networks for volumetric medical image segmentation. In 2016 Fourth International Conference on 3D Vision (3DV) (pp. 565-571). IEEE. 